\title{
Transverse olecranon osteotomy with Gigli saw: A fast and reliable technique
}

\author{
Bulent Karslioglu ${ }^{1 *}$ \\ ${ }^{1}$ PROF CEMIL TASCIOGLU CITY Hospital, DEPARTMENT OF ORTHOPEDICS AND TRAUMATOLOGY, ISTANBUL, TURKEY
}

\begin{abstract}
Objective. Distal humerus fractures constitute of approximately $2 \%$ of all fractures and $30 \%$ of elbow fractures. Olecranon osteotomy provides excellent exposure of distal humerus and articular surface. In this study, we aimed to compare transverse osteotomy with gigli saw and classical chevron osteotomy techniques in terms of osteotomy duration and clinical results. Materials and Methods. 40 elbows of 40 patients with Type B intraarticular distal humerus fractures according to AO classification were included in our study. Patients were divided into 2 groups as transverse or chevron osteotomy groups. Patients were evaluated in terms of intraoperative osteotomy time, postoperative time to union, range of motion in the elbow joint and Quick Dash scores at 6th, 12th and 24th months. Results. The mean age of the patients was 45.6 years (19-62). $40 \%$ of the patients ( 8 patients) in the Chevron group had more than $2 \mathrm{~mm}$ stepping at articular surface, while this rate was $10 \%$ ( 2 patients) in the gigli saw group. Union was obtained in all patients for both techniques. There was no significant difference between the mean QuickDASH scores and range of motion of the elbow joints except flexion in both groups. Range of motion of flexion was statistically better in the gigli saw group $(\mathrm{p}<0.05)$. Conclusions. Transverse osteotomy technique significantly reduces osteotomy and fixation time and will not cause problems in fracture union. It may be preferred because it is simpler and faster to apply than chevron osteotomy and because intra-articular stepping is less common.
\end{abstract}

Category: Original Research Paper

Received: February 17, 2021

Accepted: April 5, 2021

Published: May 10, 2021

\section{Keywords:}

distal humerus, olecranon, transverse, osteotomy

* Corresponding author:

Bulent Karslioglu,

SBU Prof. Cemil Tascioglu City Hospital, Department of Orthopedics and Traumatology, Darulaceze Street No. 25, Sisli, Istanbul, Turkey

E-mail: bukars@gmail.com

\section{Introduction}

Distal humerus fractures constitute of approximately $2 \%$ of all fractures and $30 \%$ of elbow fractures. Complex intra-articular distal humerus fractures constitute $1 \%$ of all fractures [1]. These fractures can be seen in elderly patients after simple falls due to osteoporosis and in young patients due to high energy trauma [2]. The increase in elderly population and increased usage of motor vehicles in the community reveals the fact that the number of complex distal humeral fractures that will require surgical intervention will also increase [3].

The biggest problem with these fractures, which requires anatomical reduction and fixation, is the lack of visualization of the fracture site. Olecranon osteotomy, triceps lifting, triceps splitting, and triceps sparing are the options that have been presented for to get the best results in intra-articular fractures. Most surgeons think olecranon osteotomy is mandatory and provides excellent exposure of distal humerus and articular surface. Unfortunately, the opinion about osteotomy creates controversies about the complications such as delayed union, non-union, technical difficulties in performing osteotomy, prominence of the hardware and possible risk of cartilage damage [4]. Two of the most important drawbacks are the technical difficulties of performing an osteotomy and fixation of the osteotomy to obtain a smooth joint articular face that will not cause arthrosis in the future.

In this study, we aimed to compare oblique osteotomy with gigli saw and classical chevron osteotomy techniques in terms of osteotomy duration and clinical results. There is a similar publication in the literature but we think our study is more suggestive in terms of the longer follow-up period, the higher number of cases, and the inclusion of the osteotomy completion time which may be one of the most important reasons to choose. 


\section{Materials and Methods}

Patients who underwent olecranon osteotomy for distal humerus fracture between January 2016 and January 2018 were evaluated. 40 elbows of 40 patients with Type B intraarticular distal humerus fractures according to $\mathrm{AO}$ classification were included in our study. Patients were divided into 2 groups as transverse osteotomy (TO) and chevron osteotomy (CO) groups. Random selection with sealed envelope method to ensure randomization was utilized for patients to attend surgery in one of two methods, considering the time of admission to hospital with sealed envelope method. The patient was given a pair of sealed envelopes, in which the names of the two groups were written separately, so after the first applicant selected an envelope, the next patient would be directed to a group based on the remaining envelope of the couple. After final exclusions, both groups checked and groups were found similar in terms of age, gender distribution, fracture type, and proportion with their dominant hand affected. All participants were informed, and an informed consent form was obtained. Additional informed consent was obtained from all patients for whom identifying information is included in this article. The study was approved by our Institutional Review Board.

Patients were evaluated in terms of intraoperative osteotomy time, postoperative time to union and range of motion in the elbow joint. Patients were evaluated postoperative $6^{\text {th }}, 12^{\text {th }}$ and $24^{\text {th }}$ months according to QuickDash scores.

\section{Surgical Technique}

The patient was placed in the lateral decubitus position. The operating arm placed on an arm holder. Posterior straight incision was made over the olecranon and extended proximally towards to the distal humerus. The ulnar nerve was identified within the medial intermuscular septum towards cubital tunnel and was secured with a Penrose drain. Anatomic olecranon plate was fixed with proximal and distal screws. The medial and lateral joint capsule was opened after the location of the osteotomy was determined. After osteotomy line was determined by the help of fluoroscopy, a hole wide enough for the gigli saw to enter the elbow joint was opened with a hemostat. A slightly oblique osteotomy line towards the proximal was created from the bare area of the sigmoid notch with the aid of a gigli saw (Figure 1). Distal screws belonging to the plate were removed after observing movement in the osteotomy line. In order to increase the vision to the distal humerus, the plate with the proximal osteotomy part was pulled proximally and the procedures planned for the humerus were started. After the distal humerus procedures were completed, the osteotomy line was connected using the old holes of the distal screws. Re-fixation of the plate in the same position using pre-osteotomy holes prevents stepping in the osteotomy line. The joint surface on the osteotomy line was checked again after fixation with fluoroscopy. Osteotomy line was clearly observed on postoperative radiographs (Figure 2).

Trans olecranon osteotomy with the Chevron technique was performed as described by AO [5] and after olecranon osteotomy, fixation was achieved again with an anatomical olecranon plate. A splint was applied to all patients with the elbow flexed at 70 degrees. In both groups, as long as distal humerus fixation allowed, rehabilitation was initiated for active and active-assisted flexion-extension movements in the third week. Strengthening exercises were performed on the patients until the $3^{\text {rd }}$ month after the $6^{\text {th }}$ week.

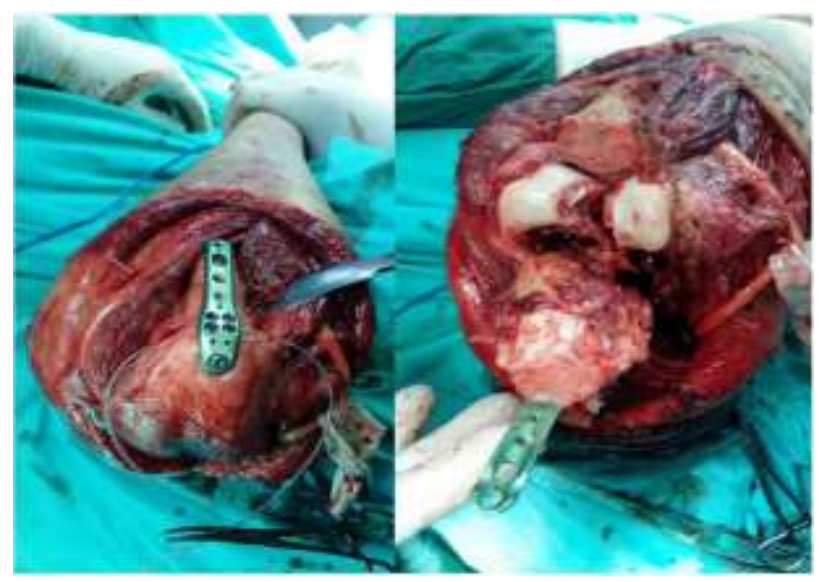

Figure 1. An oblique osteotomy was created from the bare area of the sigmoid notch with a gigli saw.

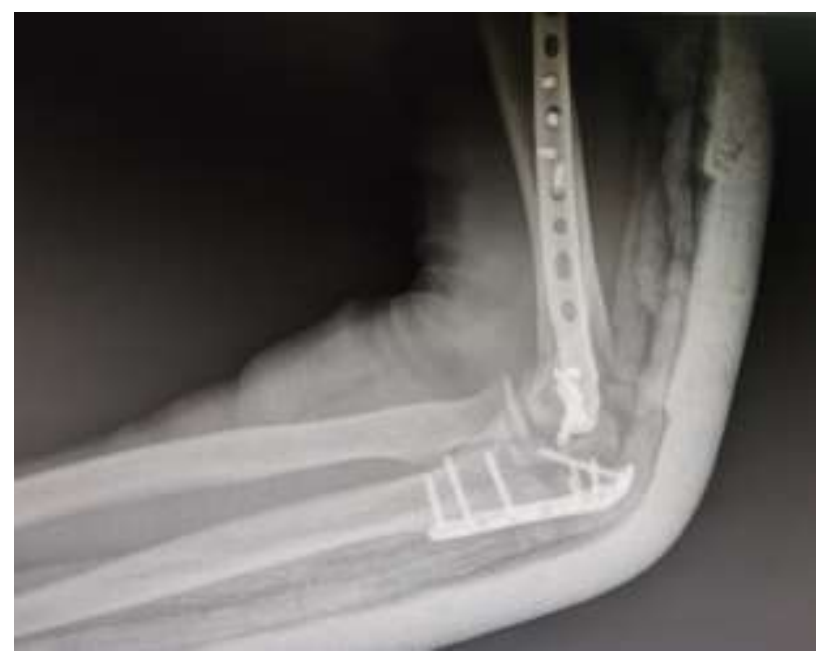

Figure 2. Osteotomy line was clearly observed on postoperative radiographs.

\section{Results}

A total of 40 patients, 20 in both groups, were included in the study. The mean age of the patients was 45.6 years (19-62). 65\% of the patients were men and $35 \%$ were women. Twenty-two (55\%) of the patients were hospitalized for fractures after falling from the stairs, 12 (30\%) from fall from ground level, and $6(15 \%)$ due to a 
motor vehicle accident. The mean follow-up time was 39.3 months (range 30-54)

Postoperative true lateral elbow radiography was performed for all patients and were evaluated in terms of articular stepping in the olecranon. Displacement of more than $2 \mathrm{~mm}$ in the articular joint was considered as stepping. According to this evaluation, $40 \%$ of the patients $(8$ patients) in the Chevron group had more than $2 \mathrm{~mm}$ stepping in the joint, while this rate was 10\% (2 patients) in the gigli saw group.

Osteotomy site bony union was evaluated radiologically at $3^{\text {rd }}$ and $6^{\text {th }}$ weeks postoperatively. Union was obtained in all patients for both techniques. There was no significant difference between the mean QuickDASH scores and range of motion of the elbow joints except flexion in both groups (Table 1).

Table 1. Statistical comparison of both groups according to the range of motion and total osteotomy time

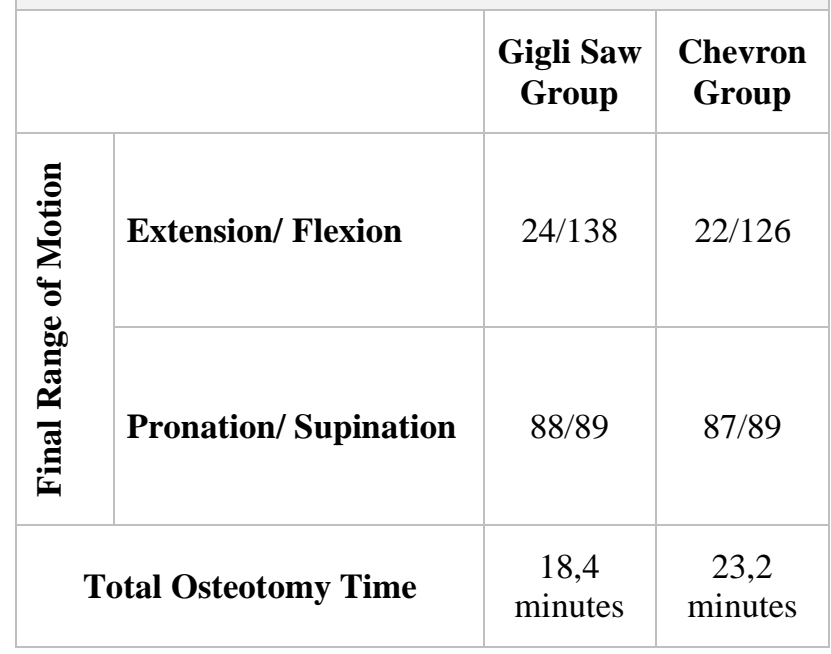

The first step was the application of all screws of the olecranon plate, followed by osteotomy with a gigli wire and then removal of the distal screws.

The second stage was the replacement of the olecranon plate and the application of the distal screws after distal humerus fracture fixation. Total osteotomy time was obtained by summing these two stages (Table 1). Range of motion of flexion was statistically better in the gigli saw group ( $\mathrm{p}=.3$ ) (Table 2).

Table 2. Comparison of the Quick Dash score for both groups by months

\begin{tabular}{|l|l|c|c|}
\hline & & $\begin{array}{c}\text { Gigli Saw } \\
\text { Group }\end{array}$ & $\begin{array}{c}\text { Chevron } \\
\text { Group }\end{array}$ \\
\hline \multirow{2}{*}{ QuickDASH } & $6^{\text {th }}$ months & 72.7 & 75.0 \\
\cline { 2 - 4 } & $12^{\text {th }}$ months & 65.9 & 68.2 \\
\cline { 2 - 4 } & $24^{\text {th }}$ months & 43.2 & 45.5 \\
\hline
\end{tabular}

\section{Discussions}

Complex intra-articular fractures which are often displaced and rotated towards proximally by the pulling of the flexor and extensor muscles, consist of approximately $1 \%$ of all fractures [1]. The increase in the number of traffic accidents has increased the frequency of high-energy and complicated fractures. Due to the increase in expected life span, fractures related to osteoporosis also increase. These fractures, Type $\mathrm{B}$ or $\mathrm{C}$ according to the $\mathrm{AO}$ classification, are intra-articular fractures that concern one or both columns. The treatment of these fractures is difficult due to the complex anatomical relationships of the distal humerus, crush injury of articular cartilage due to high energy trauma at young patients and poor bone stock due to osteoporosis at older patients. The aim must be to create anatomic intraarticular alignment and to provide stable and rigid fixation that allows early motion. In order to achieve this surgical goal, it is necessary to see the most distal and intra-articular extensions of the fracture. Olecranon osteotomy has been a preferred surgical technique for this purpose because provides clearer visualization of articular surface compared with paratricipital approaches, tricepssparing or triceps reflecting anconeus pedicle techniques $[6,7]$.

There are 2 forms of intra-articular osteotomy that can be preferred: transverse or chevron osteotomies apex distal or proximal. Chevron shaped apex distal intraarticular osteotomy is more preferred method than transverse osteotomy because chevron osteotomy theoretically may decrease bone union time and increase rotational and translational stability due to the larger bone surface and locking osteotomy fragments [8], but it is technically more difficult.

There is an anatomical field without articular cartilage called bare area in the proximal ulna. This area was originally defined by Morrey and anatomical features of this bare area was delineated by Wang et al. [1,9]. Wang et al. concluded that bare area presents at all cadaveric specimens and locate at approximately $2.1 \mathrm{~cm}$ distal from palpable tip of the olecranon. They also reported that transverse osteotomy performed $2.1 \mathrm{~cm}$ distal to the olecranon tip passed through the bare area in $67 \%$ of the samples and stated that the chance of remaining osteotomy in the bare area is higher in transverse osteotomies rather than chevron osteotomy. Wang et all. claimed that no matter how narrow the osteotomy angle, chevron osteotomy would damage the articular cartilage. We observed osteoarthritis due to chondral damage in accordance with the studies of Wang et al. at 8 patients who underwent chevron osteotomy in our study. Although physical range of motion increased in 6 patients after physical therapy, 2 patients had limited flexion. Flexion was limited in 2 patients who underwent oblique osteotomy [9]. 
Gigli saw is a low energy osteotomy and creates a neat fracture line. Continuous high-speed application of oscillating saw may cause thermal necrosis of the bone [10]. Washing the saw blade with cold saline and frequent interruption of the procedure during osteotomy may prevent this thermal necrosis. Although osteotome is used to complete the last part of the osteotomy to cracking subchondral bone, the uncontrolled use of the oscillating saw can also lead to cartilage damage. The absence of thin osteotome during osteotomy is also one of the reasons for cartilage damage. The features of Gigli saw such as being easy to find and relatively easy to use can be important for the reason for preference.

One of the most frequent and most problematic complications associated with olecranon osteotomy is delayed union and/or nonunion. According to clinical studies, the rate of nonunion after osteotomy is approximately $10 \%$ [4]. The type of osteotomy and the implant chosen for fixation are important for non-union. Transverse osteotomy has less bone contact surface, so nonunion rates are higher. Rotational and translational stability is higher due to the fragments locked in chevron osteotomy. The non-union rates are also lower due to the large contact bone surface [11]. We pulled the gigli saw distally as soon as gigli saw passes into the subchondral bone, to create an oblique rather than transverse osteotomy, to gain larger bone contact area to facilitate bony union as Ramsey et al. have described [12]. In our study, the duration of union was approximately 1.5 months at patients in whom oblique osteotomy applied with this technique. Non-union or delayed union was not observed in any of our Group 2 patients. No significant difference was found between chevron osteotomy in terms of union time.

Biomechanical studies have been carried out for many different methods for fixation after olecranon osteotomy. Tension band wiring, intramedullary screw fixation or anatomic locking plates can be selected for fixation purposes. Studies have showed that locking plates provide more compression force than other 2 fixation options. It has been reported that the risk of implant failure is higher in tension band wiring $[13,14]$. In our study, we used an anatomically locked olecranon plate for both groups. Implant failure or hardware prominence requiring implant removal was not observed in any of our patients.

There is no definite consensus on the location and angle of chevron osteotomy. Jupiter et all [15] and Muller et all [16] have suggested making trochlea level, Henley et all [17] recommended $3 \mathrm{~cm}$ proximal from the olecranon tip, Wang et al. [9] recommended $2.1 \mathrm{~cm}$ proximal from olecranon tip, Nauth et al. [18] suggested 2.5 to $3 \mathrm{~cm}$ from the tip of olecranon. It is very difficult to try to make osteotomy, where millimeter measurements are important to prevent damage to the cartilage, from a $4.11 \mathrm{~mm}$ wide bare area according to Kieffer et al. [19]. Although all these studies try to determine the anatomical starting point for
Chevron osteotomy, there is no descriptive explanation regarding the angle between the osteotomy legs. Choosing an easier and one-dimensional oblique osteotomy will prevent cartilage damage because bare area is much smaller than the chevron osteotomy [9].

It is important to perform rigid and anatomical fixation in the osteotomy line and not to cause step-off. Ramsey et al. have described a new technique that allows anatomical fixation of osteotomy fragments [12]. According to this technique the anatomic olecranon plate is fixed to the bone by applying distal and proximal screws before starting osteotomy. We used gigli saw to create osteotomy approximately $2.1 \mathrm{~cm}$ from the tip of the triceps insertion point entering into the bare area with taking into account the cortical notch, if any. The cortical notch is an anatomical protrusion that is not seen in all patients, but its presence indicates the location of the bare area. Distal screws were pulled out after osteotomy. Proximal osteotomy fragment with plate was removed from the osteotomy area towards proximally, allowing complete visualization of the fracture and joint. We achieved anatomical and complete union in osteotomies with this technique, due to the plate was applied before osteotomy and distal fixation was made from the same screw holes after osteotomy.

The most important problem with oblique osteotomy will be the time and effort to avoid stepping off the joint. The shape of the V-shaped osteotomy that allows the locking of the fragments in Chevron osteotomy has advantages of minimizing the step-off in the joint and allowing rapid fixation. In our study, we found oblique osteotomy with gigli saw applying anatomical plate before reduced intra-articular step off and prevented time lost during osteotomy process. When considering comparison of osteotomy and fixation times for both groups, total time was significantly lower in the oblique osteotomy group.

\section{Conclusions}

We found that transverse osteotomy technique significantly reduces osteotomy and fixation time and will not cause problems in fracture union. It may be preferred because it is simpler and faster to apply than chevron osteotomy and because the intra-articular stepping is less common.

\section{Conflict of interest disclosure}

There are no known conflicts of interest in the publication of this article. The manuscript was read and approved by all authors.

\section{Compliance with ethical standards}

Any aspect of the work covered in this manuscript has been conducted with the ethical approval of all relevant bodies and that such approvals are acknowledged within the manuscript. 


\section{References}

1. Civan O, Ugur L, Yildiz G. Comparison of two surgical techniques for the treatment of transverse olecranon fractures: A finite element study. Int J Med Robot. 2021 Apr 14:e2265. doi: 10.1002/rcs.2265.

2. Anglen J. Distal humerus fractures. J Am Acad Orthop Surg. 2005;13(5):291-7. doi: 10.5435/00124635200509000-00001

3. Palvanen M, Niemi S, Parkkari J, Kannus $P$. Osteoporotic fractures of the distal humerus in elderly women. Ann Intern Med. 2003;139(3):W-W61. doi: 10.7326/0003-4819-139-3-200308050-00021-w2

4. McKee MD, Wilson TL, Winston L, Schemitsch EH, Richards RR. Functional outcome following surgical treatment of intra-articular distal humeral fractures through a posterior approach. J Bone Joint Surg Am. 2000;82(12):1701-7. doi: 10.2106/00004623200012000-00003

5. Buckley RE, Moran CG, Apivatthakakul T. AO Principles of Fracture Management. Vol. 1: Principles, Vol. 2: Specific fractures. 2018 Third Edition Thieme Verlagsgruppe, Stuttgart, New York, Delhi, Rio. Online ISBN: 9783132423107, doi: 10.1055/b-006149767

6. Wilkinson JM, Stanley D. Posterior surgical approaches to the elbow: a comparative anatomic study. J Shoulder Elbow Surg. 2001;10(4):380-2. doi: 10.1067/mse.2001.116517

7. Elmadag M, Erdil M, Bilsel K, Acar MA, Tuncer N, Tuncay I. The olecranon osteotomy provides better outcome than the triceps-lifting approach for the treatment of distal humerus fractures. Eur $J$ Orthop Surg Traumatol. 2014;24(1):43-50. doi: 10.1007/s00590-012-1149-y

8. Gainor BJ, Moussa F, Schott T. Healing rate of transverse osteotomies of the olecranon used in reconstruction of distal humerus fractures. J South Orthop Assoc. 1995;4(4):263-8.

9. Wang AA, Mara M, Hutchinson DT. The proximal ulna: An anatomic study with relevance to olecranon osteotomy and fracture fixation. J Shoulder Elbow Surg. 2003;12(3):293-6. doi: 10.1016/s10582746(02)86803-3
10. Dabis J, Templeton-Ward O, Lacey AE, Narayan B, Trompeter A. The history, evolution and basic science of osteotomy techniques. Strategies Trauma Limb Reconstr. 2017;12(3):169-180. doi: 10.1007/s11751017-0296-4

11. Ring D, Gulotta L, Chin K, Jupiter JB. Olecranon osteotomy for exposure of fractures and nonunions of the distal humerus. J Orthop Trauma. 2004;18(7):4469. doi: 10.1097/00005131-200408000-00010

12. Ramsey DC, Thompson AR, Nazir OF, Mirarchi AJ. A new technique for olecranon osteotomy in the treatment of distal humeral fractures. JSES Open Access. 2018;3(1):1-4. doi: 10.1016/j.jses.2018.09.001

13. Wilson J, Bajwa A, Kamath V, Rangan A. Biomechanical comparison of interfragmentary compression in transverse fractures of the olecranon. $J$ Bone Joint Surg Br. 2011;93(2):245-50. doi: 10.1302/0301-620X.93B2.24613

14. Hahn A, O'Hara NN, Koh K, Zhang LQ, O'Toole RV, Andrew Eglseder W. Is intramedullary screw fixation biomechanically superior to locking plate fixation and/or tension band wiring in transverse olecranon fractures? A cadaveric biomechanical comparison study. Injury. 2020;51(4):850-855. doi: 10.1016/j.injury.2020.02.059

15. Jupiter JB, Neff U, Holzach P, Allgöwer M. Intercondylar fractures of the humerus. An operative approach. J Bone Joint Surg Am. 1985;67(2):226-39.

16. Müller ME, Allgöwer M, Schneider R, Willenegger $H$. Manual of internal fixation: techniques recommended by the AO-ASIF group. Springer Science \& Business Media; 1991. ISBN 978-3-662-02695-3

17. Henley MB, Bone LB, Parker B. Operative management of intra-articular fractures of the distal humerus. J Orthop Trauma. 1987;1(1):24-35. doi: 10.1097/00005131-198701010-00004

18. Nauth A, McKee MD, Ristevski B, Hall J, Schemitsch EH. Distal humeral fractures in adults. $J$ Bone Joint Surg Am. 2011;93(7):686-700. doi: 10.2106/JBJS.J.00845

19. Kieffer EM, Bouchaib J, Bierry G, Clavert P. CT arthrography and anatomical correlation of the bare area of the ulnar trochlear fossa: a risk of misdiagnosis of cartilage ulcerations. Surg Radiol Anat. 2014;36(5):481-6. doi: 10.1007/s00276-013-1200-7 